\title{
A new superclass of block backward differentiation formula for stiff ordinary differential equations
}

\begin{abstract}
A superclass of block backward differentiation formula (BBDF) suitable for solving stiff ordinary differential equations is developed. The method is of order 3 , with smaller error constant than the conventional BBDF. It is A-stable and generates two points at each step of the integration. A comparison is made between the new method, the 2-point block backward differentiation formula (2BBDF) and 1-point backward differentiation formula (1BDF). The numerical results show that the method developed outperformed the 2BBDF and 1BDF methods in terms of accuracy. It also reduces the integration steps when compared with the 1BDF method.
\end{abstract}

Keyword: Implicit block method; A-stability; Superclass of block backward differentiation formula; Stiff ordinary differential equations 